\title{
Smartphone applications to aid weight loss and management: current perspectives
}

This article was published in the following Dove Press journal:

Diabetes, Metabolic Syndrome and Obesity:Targets and Therapy 18 July 2016

Number of times this article has been viewed

\section{Elizabeth F Sutton \\ Leanne M Redman}

Pennington Biomedical Research Center, Louisiana State University, Baton Rouge, LA, USA
Correspondence: Leanne M Redman Pennington Biomedical Research Center, Louisiana State University, 6400 Perkins Road, Baton Rouge, LA 70808, USA

Tel + I 2257630947

Fax +I 2257633009

Email Leanne.Redman@pbrc.edu
Abstract: The development and dissemination of smart devices has cultivated a global environment of hyperconnectivity and increased our access to information. The paralleled launch and success of the Mobile Health industry has created a market of commercially available applications or "apps" along with tools or sensors, which allow the user to receive and collect personal health information. Apps and accompanying tools now allow an individual to "self-digitize" and, pertaining to weight management, monitor their body weight, caloric intake, physical activity, and more. These products possess the ability to improve the scalability of traditional in-person weight management services considering their near ubiquity, affordability, and capability to deliver information directly and personally to the user. However, similar to the dietary supplement market, the anecdotal value of these products has driven their popularity and acceptance by the general public without requirement of scientific validation or, in the area of weight management or diet/exercise, validation of the safety and efficacy by the Food and Drug Administration prior to market launch. By conducting a literature and clinical trial search, we found remarkably few active, completed, or published studies testing the efficacy of smart device applications using randomized controlled trials. Research efforts must be focused on illuminating the efficacy of behavioral interventions and remote self-monitoring for weight loss/maintenance treatment with true, randomized controlled trials.

Keywords: smartphone, mobile phone, application, app, weight, weight loss, weight maintenance

\section{Discussion}

Smart devices are nearing ubiquity. Since their development, these devices continue to increase the local and global connectivity of people and improve our access to information. In 2015, there was an estimated 3.5 devices for every human on earth, and it is predicted that there will be a doubling of Internet-enabled mobile devices to 50 billion devices by 2020 (6.6 devices per person). ${ }^{1}$ In conjunction with the creation of smart devices has been the birth of commercial markets to complement these devices, including the development of mobile applications or "apps", as well as tools and sensors intended to be operated through or paired with the device. The Mobile Health, or mHealth, industry holds a large portion of this market, and the demand for improvements in individual health behaviors has driven this market forward with the development of apps to assist in health improvement. Furthermore, the desire of humans to "self-digitize" has driven the development of internal sensors and remote tools for tracking of health and behaviors. Remote tools, including pedometers, accelerometers, bathroom scales, pill bottles, and blood pressure cuffs, are paired with these devices through apps to allow the user to interpret their own data and self-monitor health behaviors such as tracking 
of food intake, physical activity, body weight, sleep quality and duration, blood sugar, and blood pressure (Figure 1). These products operate through the collection and transmission of empirical data to the smart device, which allows for real-time, objective self-monitoring. The more sophisticated apps are able to interpret these data and provide near real-time feedback to the user.

Our global population is connected and engaged in an ideal environment for dissemination of information because of the increased availability of Internet-enabled devices and rapid development of complementary products (apps, sensors, and tools). Indeed, these devices present opportunity to build upon the scalability, feasibility, and efficacy of traditional in-person treatment for weight loss or maintenance. For example, in the US, more than two-thirds of adults have a body mass index $>25 \mathrm{~kg} / \mathrm{m}^{2}$ and $>140$ million of these adults are eligible for weight loss treatment. ${ }^{2}$ While the gold standard for weight loss treatment is an in-person approach (14 in-person contacts over 6 months) with personalized goals, advice, and feedback, ${ }^{3}$ the Academy of Nutrition and Dietetics reports only 94,671 registered dietitian nutritionists in the US as of February $2016 .{ }^{4}$ If all registered dietitian nutritionists were exclusively

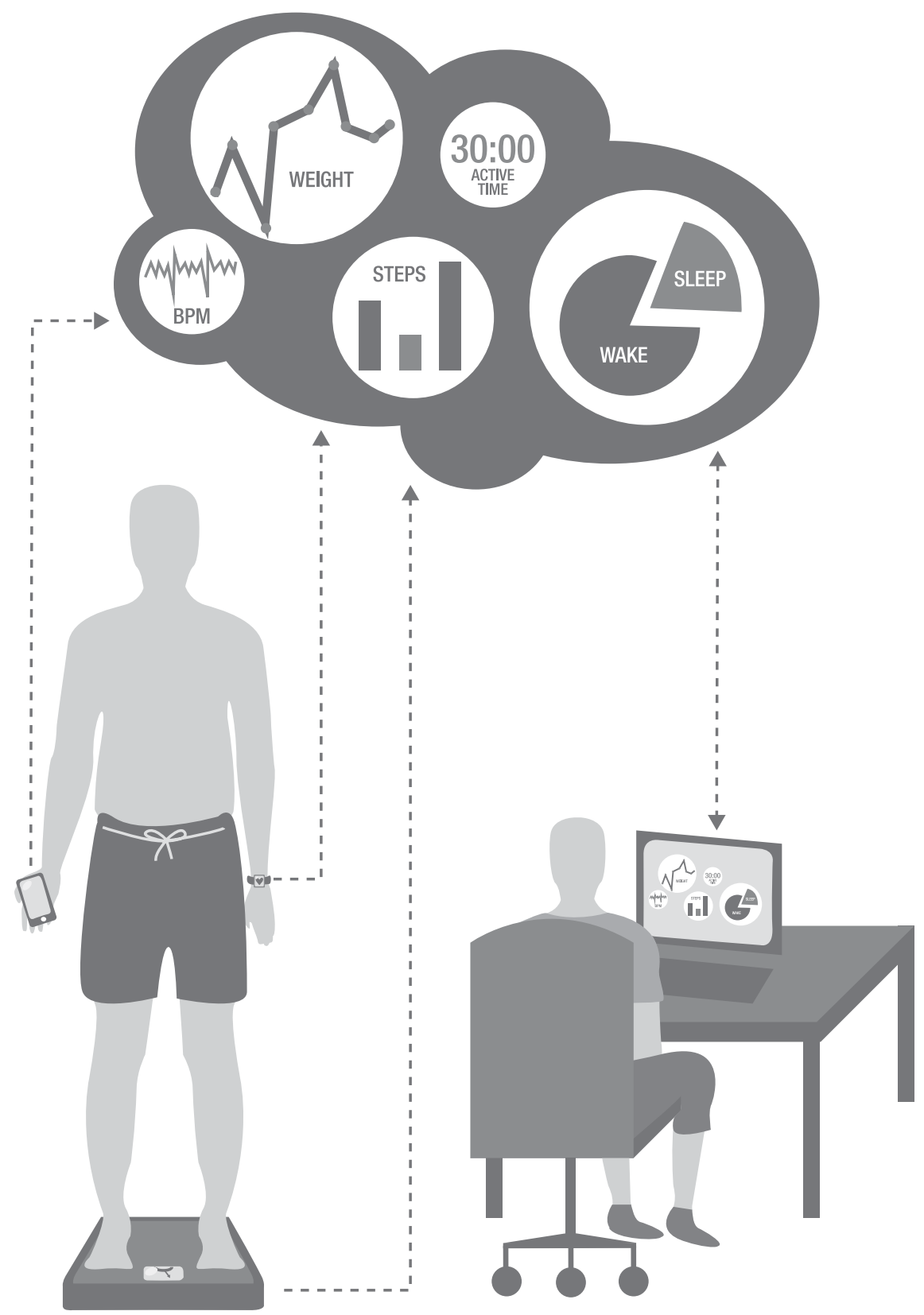

Figure I Representation of individual self-monitoring through smart device applications, tools, and sensors (e.g. weight, activity, steps per day, heart rate (BPM) and sleep time) for personal information or patient/counselor data sharing.

Abbreviation: BPM, beats per minute. 
working to deliver weight loss treatment, this would yield a patient:provider ratio of $\sim 1,500: 1$, suggesting that the inperson model for implementing weight loss treatment to all individuals is currently grossly inadequate. Hence, the ability to increase scalability of weight management programs is an obvious benefit of a smart device-based model compared to the traditional, in-person model. Reduction in cost and time for both patient and provider supports the feasibility of smart device-driven models. Apps disseminating smoking cessation interventions have extensively demonstrated that apps have the ability to serve as a platform for delivery of behavioral interventions. ${ }^{5}$ In fact, mHealth technology allows for implementation of behavior change therapies because it retains the principles with which in-person treatment derives success: frequent engagement, consistency over time, and feedback on behavior. Provision of the near real-time feedback that mHealth interventions allow supports the potential for the efficacy of smart device models to be equivalent to the inperson model. Indeed, computer code can be easily developed to interpret user data and deliver automated near real-time feedback from providers congruent with patient data collected via these tools (Figure 1). For example, tracking food intake or recording number of steps/day using a pedometer has been shown in clinical trials to produce more successful weight and behavior change. ${ }^{6-8}$ Although apps have been shown to be capable of delivering behavior change theories, reviews of content analysis of commercially available physical activity and weight management apps have shown very poor inclusion of behavioral change theories. ${ }^{9,10}$ In fact, in a review of 204 commercially available weight loss apps, none of the apps reviewed included all 13 evidence-informed practices for weight loss the authors identified common to the Centers for Disease Control and Prevention (CDC), National Institutes of Health (NIH), Food and Drug Administration (FDA), and US Department of Agriculture (USDA): 1) assess one's weight, 2) eat a diet rich in fruits and vegetables, 3) perform regular physical activity, 4) drink water instead of juice or soda, 5) keep a food diary, 6) maintain calorie balance of in versus out, 7) weight loss of 1-2 lb/wk, 8) portion control, 9) read nutrition labels, 10) track your weight, 11) keep a physical activity journal, 12) plan meals, and 13) seek social support. ${ }^{11}$ Only $21 \%$ of apps studied recommended regular physical activity, $6 \%$ recommended a weight loss goal of $1-2$ pounds/ wk, and only $36 \%$ of these apps even assessed the user's weight at all (ie, calculated the user's body mass index). ${ }^{11}$ It also remains to be determined whether behavioral economics in favor of the patient and provider are also realized by the increased use of smartphones in health care delivery system. ${ }^{12}$
The mHealth market is booming, but is the science keeping up? Evaluated at $\$ 10.5$ billion in 2014 , this market is projected to reach $\sim \$ 60$ billion by 2020 with a compound annual growth rate of $>30 \% .{ }^{13}$ As the mHealth market continues to grow, smart devices and their associated apps, sensors, and tools are increasingly available at progressively affordable costs. As of February 11, 2016, >60,000 "Health \& Fitness" apps were commercially available to Android consumers (Android accounts for an estimated 50\% of the mobile device market) ${ }^{14}$ Additionally, demonstrating the growth of market for tools, Fitbit Inc. (a company that produces wearable fitness trackers founded in 2007) reported an increased revenue from $\$ 745$ million in 2014 to $\$ 1.858$ billion in 2015 . $^{15}$

Similar to the dietary supplement market, the anecdotal value of these products has driven their popularity and acceptance by the general public without requirement of scientific validation or, in the area of weight management or diet/ exercise, approval by the FDA prior to market launch. To this point, a search of clinicaltrials.gov (accessed February 5, 2016) using terms "smartphone, mobile phone, app, application, and weight" identified 56 registered research studies, of which only 16 were randomized controlled trials (RCTs) testing weight loss/maintenance programs delivered by smart device application compared to either no intervention or in-person intervention. ${ }^{16} \mathrm{~A}$ search of PubMed.gov (accessed February 5,2016 ) with the same keywords (smartphone, mobile phone, app, application, and weight, filters: English and humans) produced 65 search results, of which only six citations were a) RCTs and b) testing the efficacy of smart device application. ${ }^{17}$ Perhaps, it is the ubiquity of these devices and their products, which has led much of the scientific community to trust the very few RCTs supporting the efficacy of delivering interventions remotely via apps. Many clinical trials in fact have incorporated common commercially available applications to aid in data collection or intervention support (e.g., Lose It! to track food intake), while RCTs testing the efficacy of these apps are still lacking. Consequently, there is an increasingly large gap between production and dissemination of products and proof of their efficacy and safety. The speedy developmentto-dissemination rate of technology can be intimidating for researchers and can impede the ability for appropriate testing of effectiveness. An app that winds up being tested in an RCT will likely be outdated by the time the results are published and even more outdated before it can be integrated into clinical practice. However, it does not negate the necessity for validation. Perhaps, testing general principles, for example, the inclusion of common evidence-informed practices or implementation of behavior change strategies, delivered remotely through apps 
versus the in-person model may be a more advantageous path to determine the efficacy of these products.

We are living within a hyperconnected new age of technology, where smart devices and smart device products are nearly ever-present, and the vast majority of the world population has immediate access to the Internet. Simultaneously, we are arguably living among the sickest and heaviest population yet, and the in-person model for dissemination of weight loss/maintenance programs is unsustainable to successfully reach the world's health goals. ${ }^{18}$ Considering the potential aforementioned benefits, implementing weight management programs using smart devices could potentially lessen health care system costs and burden (as well as the burden of the patient), increase scalability of dissemination, and improve efficacy of a program, but there is a dire need of controlled RCTs and published peer-reviewed data. Research efforts must be focused on illuminating the efficacy of behavioral interventions and remote self-monitoring for weight loss/maintenance treatment with true RCTs.

\section{Disclosure}

The authors report no conflicts of interest in this work.

\section{References}

1. Topol EJ, Steinhubl SR, Torkamani A. Digital medical tools and sensors. JAMA. 2015;313(4):353-354.

2. Stevens J, Oakkar EE, Cui Z, Cai J, Truesdale KP. US adults recommended for weight reduction by 1998 and 2013 obesity guidelines, NHANES 2007-2012. Obesity (Silver Spring). 2015;23(3):527-531.

3. Jensen MD, Ryan DH, Apovian CM, et al. 2013 AHA/ACC/TOS guideline for the management of overweight and obesity in adults: a report of the American College of Cardiology/American Heart Association Task Force on Practice Guidelines and The Obesity Society. Circulation. 2014;129(25 suppl 2):S102-S138.
4. Commission on Dietetic Registration [webpage on the Internet]. Registry Statistics. Available from: https://www.cdrnet.org/registry-statistics. Accessed February 4, 2016.

5. Backinger CL, Augustson EM. Where there's an app, there's a way? Am J Prev Med. 2011;40(3):390-391.

6. Schultz W. Behavioral theories and the neurophysiology of reward. Annu Rev Psychol. 2006;57:87-115.

7. Burke LE, Wang J, Sevick MA. Self-monitoring in weight loss: a systematic review of the literature. J Am Diet Assoc. 2011;111(1): 92-102.

8. Burke LE, Conroy MB, Sereika SM, et al. The effect of electronic selfmonitoring on weight loss and dietary intake: a randomized behavioral weight loss trial. Obesity (Silver Spring). 2011;19(2):338-344.

9. Cowan LT, Van Wagenen SA, Brown BA, et al. Apps of steel: are exercise apps providing consumers with realistic expectations? A content analysis of exercise apps for presence of behavior change theory. Health Educ Behav. 2013;40(2):133-139.

10. Azar KM, Lesser LI, Laing BY, et al. Mobile applications for weight management: theory-based content analysis. Am J Prev Med. 2013;45(5): 583-589.

11. Breton ER, Fuemmeler BF, Abroms LC. Weight loss-there is an app for that! But does it adhere to evidence-informed practices? Transl Behav Med. 2011;1(4):523-529.

12. Loewenstein G, Asch DA, Volpp KG. Behavioral economics holds potential to deliver better results for patients, insurers, and employers. Health Aff (Millwood). 2013;32(7):1244-1250.

13. Dhabale S. World mHealth Market - Opportunities and Forecasts, 2014 - 2020. Portland, OR: Allied Market Research; 2015.

14. AppBrain [webpage on the Internet]. Most popular Google Play Categories. Available from: http://www.appbrain.com/stats/androidmarket-app-categories. Accessed February 11, 2016.

15. Fitbit Inc. Fitbit Fourth Quarter and Full Year 2015 Financial Summary. San Francisco, CA: Fitbit Inc; 2016.

16. ClinicalTrials.gov [homepage on the Internet]. National Library of Medicine. Available from: www.clinicaltrials.gov. Accessed February 5, 2016.

17. PubMed [homepage on the Internet]. U.S. National Library of Medicine. Available from: www.pubmed.org. Accessed February 5, 2016.

18. Office of Disease Prevention and Health Promotion [webpage on the Internet]. 2020 Topics and Objectives: Nutrition and Weight Status. Available from: http://www.healthypeople.gov/2020/topics-objectives/ topic/nutrition-and-weight-status/objectives. Accessed February 15, 2016.
Diabetes, Metabolic Syndrome and Obesity: Targets and Therapy is an international, peer-reviewed open-access journal committed to the rapid publication of the latest laboratory and clinical findings in the fields of diabetes, metabolic syndrome and obesity research. Original research, review, case reports, hypothesis formation, expert opinion and commentaries are all considered for publication. The manuscript management system is completely online and includes a very quick and fair peer-review system, which is all easy to use. Visit http://www.dovepress.com/testimonials.php to read real quotes from published authors. 